\section{Niña lactante con estenosis traqueal y arteria pulmonar izquierda en hamaca}

\section{Tracheal stenosis in an infant with sling pullmonar}

Pérez-Juárez Fabiola ${ }^{1}$, Alemán-Sánchez Natalia² ${ }^{2}$ López-Corella Eduardo ${ }^{2}$

\section{RELATO DEL CASO}

Niña de 3 meses y 16 días de edad que acudió al Servicio de Urgencias del Instituto Nacional de Pediatría (Ciudad de México) con un cuadro respiratorio de rápida evolución y falleció horas después.

Antecedentes relevantes: el embarazo fue normoevolutivo y la madre tomó ácido fólico a partir del segundo trimestre. La paciente nació por vía abdominal por presentación transversa a las 39 semanas de gestación. Apgar de 7/9, peso de 2,826 g y talla de $49 \mathrm{~cm}$. Recibió reanimación neonatal básica y oxígeno suplementario y egresó a las 72 horas.

Desde el nacimiento se le notó microtia izquierda y a la edad de 2 meses se le diagnosticó agenesia pulmonar, sin precisarse las circunstancias en las que se hizo el diagnóstico. El núcleo familiar incluía ambos padres y tres hermanos, todos sanos.

La niña se refería como asintomática hasta dos días antes de su ingreso, cuando empezó con tos productiva, rinorrea hialina, fiebre no cuantificada y rechazo al alimento. Al día siguiente presentó dificultad respiratoria con tiros intercostales y polipnea. Al interrogatorio dirigido se determinó que había estado cianótica desde el nacimiento.
${ }^{1}$ Servicio de Cardiología

${ }^{2}$ Departamento de Patología.

Instituto Nacional de Pediatría, Ciudad de México.

Recibido: 17 de enero del 2017

Aceptado: 30 de enero del 2017

Correspondencia

Dra. Fabiola Pérez-Juárez

pj_fabi@hotmail.com

Este artículo debe citarse como

Pérez-Juárez F, Alemán-Sánchez N, López-Corella E. Niña lactante con estenosis traqueal y arteria pulmonar izquierda en hamaca. Acta Pediatr Mex. 2017;38(2):108-115

DOI: http://dx.doi.org/10.18233/APM38No2pp108-1151362 
$\mathrm{Al}$ ingreso se encontraba febril, con temperatura de 39.8 grados centígrados, con taquicardia de 200 latidos por minuto y frecuencia respiratoria de 70 respiraciones por minuto. Pesaba $4 \mathrm{~kg}$, con talla de $51 \mathrm{~cm}$ y perímetro cefálico de $39 \mathrm{~cm}$. La tensión arterial era de 91/60 mmHg. Estaba somnolienta, reactiva a estímulos y bien hidratada. Se confirmó la microtia izquierda y se describió microrretrognatia con macroglosia y difícil exploración de glotis. Presentaba cianosis central generalizada, narinas permeables sin secreción y en tórax se apreciaban tiros intercostales, subcostales, retracción xifoidea, supraesternal, hipoventilación y rudeza respiratoria en hemitórax derecho; estertores y sibilancias en el izquierdo. Los ruidos cardiacos eran rítmicos y de adecuada intensidad, situados a la derecha. El hígado se palpaba $1 \mathrm{~cm}$ por debajo del reborde costal y el Ilenado capilar en extremidades era de 4 segundos.

La radiografía de tórax a su ingreso mostró ausencia de pulmón derecho con radio opacidad de todo el hemitórax derecho y con sobredistensión pulmonar izquierda compensatoria; y no se visualizaba la silueta cardiaca (Figura 1).

Las pruebas de laboratorio reportaron $\mathrm{Hb} 13.9 \mathrm{~g} /$ dL, Hto 42.3\%, leucocitos 17,400 células/mcL con 11,500 neutrófilos/mcL, 5,600 linfocitos/ mcL y plaquetas de 409,000 plaquetas/mcL. Se instaló tratamiento con penicilina G sólida cristalina a 300,000 U/kg/día y recibió salbutamol, $0.15 \mathrm{mg} / \mathrm{kg} /$ do por sibilancias. Dada la insuficiencia respiratoria se decidió intubación endotraqueal; se logró intubar al octavo intento y en la exploración endoscópica se visualizó estenosis concéntrica de la tráquea a nivel del tercer anillo.

El ecocardiograma transtorácico informó dextrocadia con levoapex, hipertensión arterial pulmonar suprasistémica, dilatación de cavidades derecha, así como interrupción de la vena

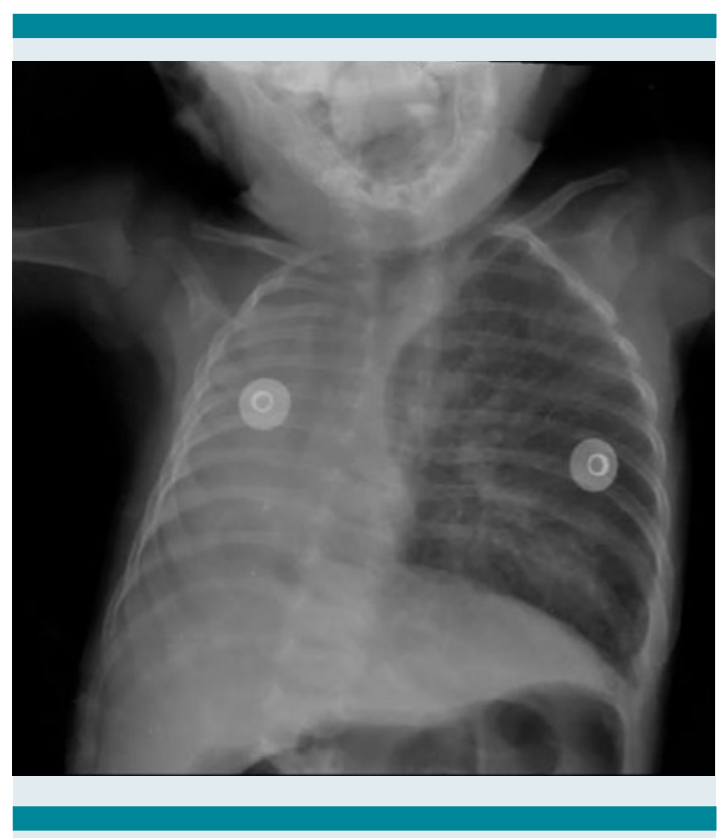

Figura 1. Radiografía de tórax que muestra ausencia de pulmón derecho y de su vasculatura. Pulmón izquierdo normal con hiperinsuflación.

cava inferior con continuación por la hemiácigos. El conducto arterioso estaba permeable de $6 \mathrm{~mm}$ de diámetro interno, con cortocircuito de derecha a izquierda (Figura 2).

En las horas siguientes la niña evolucionó mal, con retención persistente de $\mathrm{CO}_{2}$, sin lograr mantener la ventilación. Se realizó punción pleural sin recuperación, presentó asistolia que no respondió a maniobras de reanimación cardiovascular y falleció a las 24 horas de su ingreso. Los diagnósticos clínicos de defunción fueron: insuficiencia respiratoria, neumonía bacteriana, cardiopatía congénita cianógena compleja, agenesia pulmonar derecha.

\section{COMENTARIO ANATOMOPATOLÓGICO}

La autopsia en esta niña extendió y detalló el espectro de sus anomalías congénitas; (Figura 3) además de confirmar la microtia izquierda con 


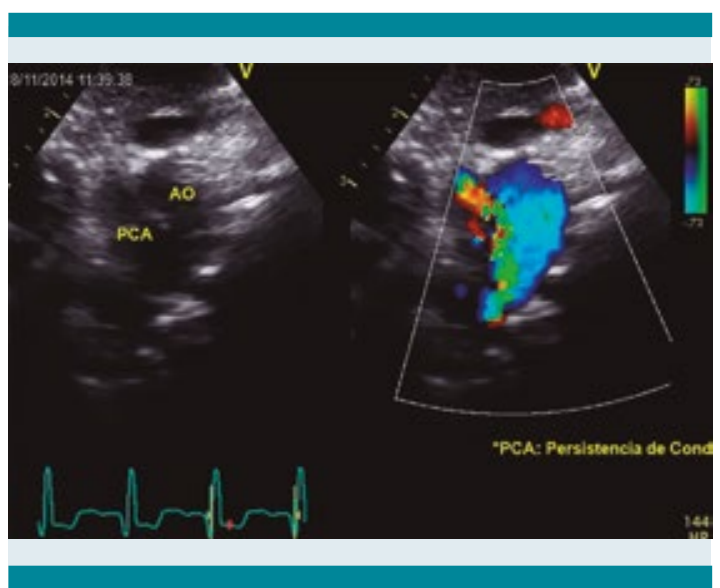

Figura 2. Corte supraesternal donde se visualiza conducto arterioso permeable (PCA).

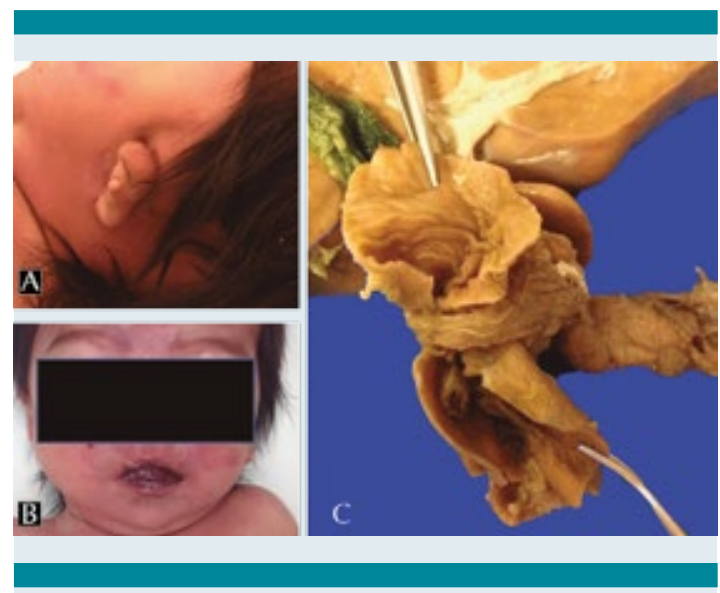

Figura 3. En el aspecto externo era evidente la microtia izquierda (A) y la microrretrognatia (B). Un páncreas anular rodea al duodeno $(\mathbf{C})$.

implantación baja de pabellón auricular derecho y la retrognatia que tanto dificultó la intubación, nos reveló un páncreas anular que rodeaba el duodeno, una gran cantidad de tejido tímico junto a las paratiroides, restos glandulares de tipo salival en hipófisis y un pulmón izquierdo conformado por un solo lóbulo, con un esbozo de língula. Pero lo que determinó la fatal evolución de la paciente es una compleja malformación que afectó el dominio cardiopulmonar.
En el tórax destacó un pulmón derecho marcadamente hipoplásico; el corazón era grande y ocupaba la cavidad torácica derecha (Figura 4). No se identificó la vena cava inferior y las vena suprahepáticas desembocaban directamente en la aurícula derecha. El drenaje del territorio de la vena cava inferior se hacía por una hemiácigos a la vena cava superior. La aurícula derecha era normal, la válvula tricúspide tuvo una valva anterior hipoplásica que le daba un aspecto bivalvo y se identificó un trombo pequeño reciente, adherido a la valva posterior. La arteria pulmonar estaba dilatada, con un conducto

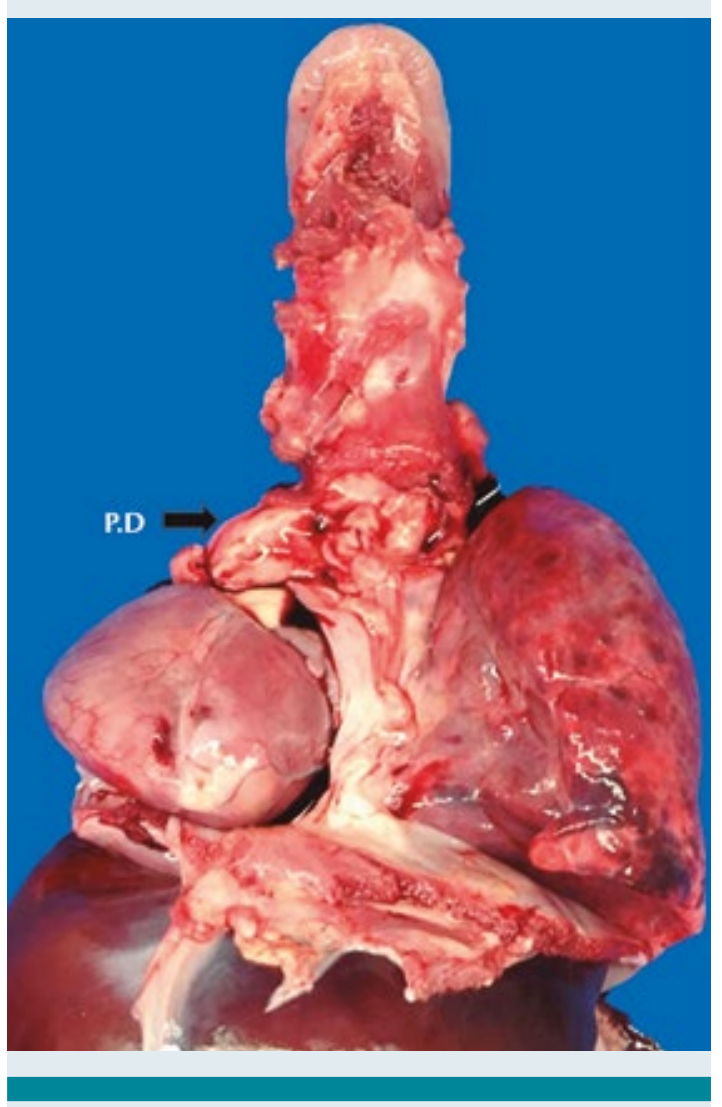

Figura 4. Aspecto anterior tóraco-abdominal. El pulmón izquierdo no tiene cisuras interlobares y se aprecia un esbozo de língula. El pulmón derecho (PD) es muy pequeño. El corazón ocupa el hemitórax derecho. 
arterioso permeable y amplio y con una rama izquierda grande que se insinuaba por detrás de la tráquea, entre ésta y el esófago ("arteria pulmonar izquierda en hamaca") con compresión de la pared posterior de la tráquea (Figura 5). La arteria pulmonar derecha era delgada y llegaba a un pulmón derecho muy pequeño (Figura 6). La aurícula izquierda recibía las venas pulmonares izquierdas de aspecto normal y una vena pulmonar derecha filiforme (Figura 7). Había una comunicación interventricular puntiforme perimembranosa.

La tráquea tenía un aspecto de embudo con estenosis de su calibre distal y cerca de la carina en su cara posterior se apreciaba una profunda depresión por compresión extrínseca de la arteria pulmonar izquierda, merced al trayecto aberrante retrotraqueal de la arteria que se insinuaba entre la tráquea y el esófago (la arteria pulmonar izquierda en hamaca o sling left pulmonary artery) (Figura 8).

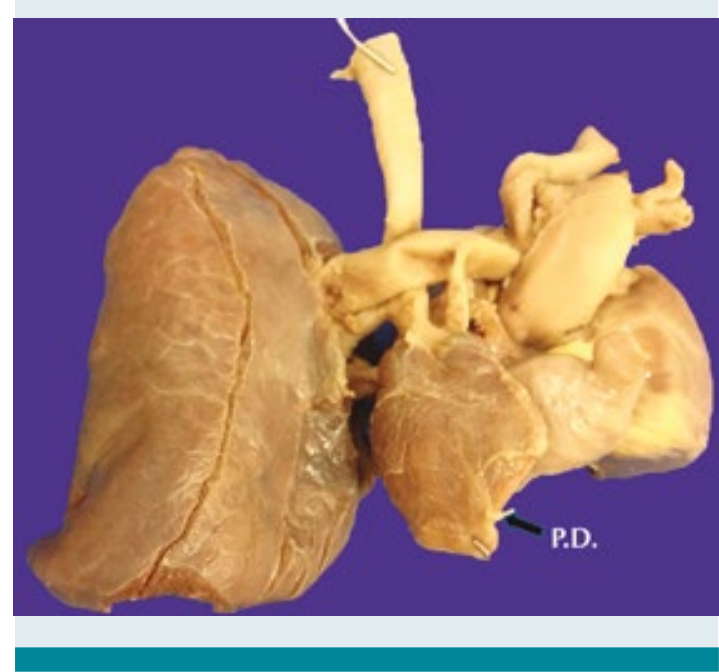

Figura 5. Aspecto posterior tóraco-abdominal. Pulmón izquierdo no lobulado. Pulmón derecho (PD) hipoplásico. Una gruesa arteria pulmonar izquierda pasa por detrás de la tráquea y la comprime. La arteria pulmonar derecha es delgada y parece emerger de la izquierda.

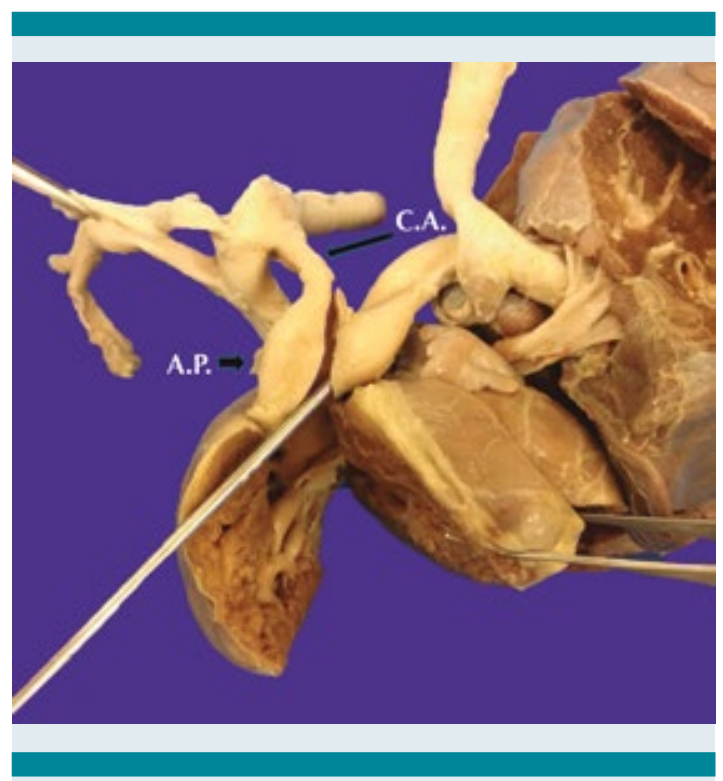

Figura 6. Vista anterior. El tronco de la pulmonar está dilatado (AP) con su rama izquierda retrotraqueal. El conducto arterioso (CA) es permeable.

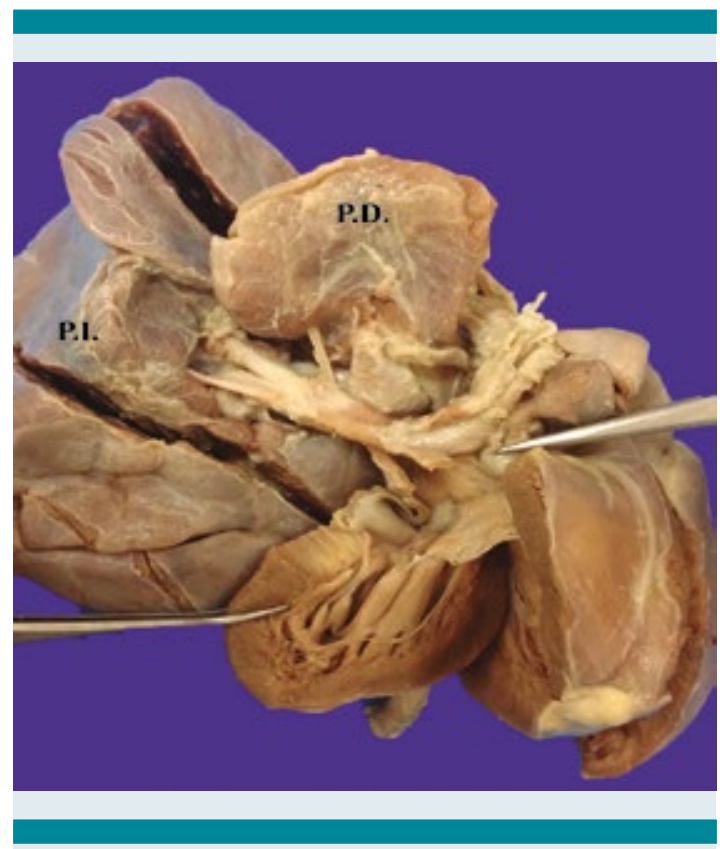

Figura 7. Dos venas pulmonares izquierdas y una derecha muy delgada drenan normalmente a la aurícula derecha (PI: pulmón izquierdo; PD: pulmón derecho). 


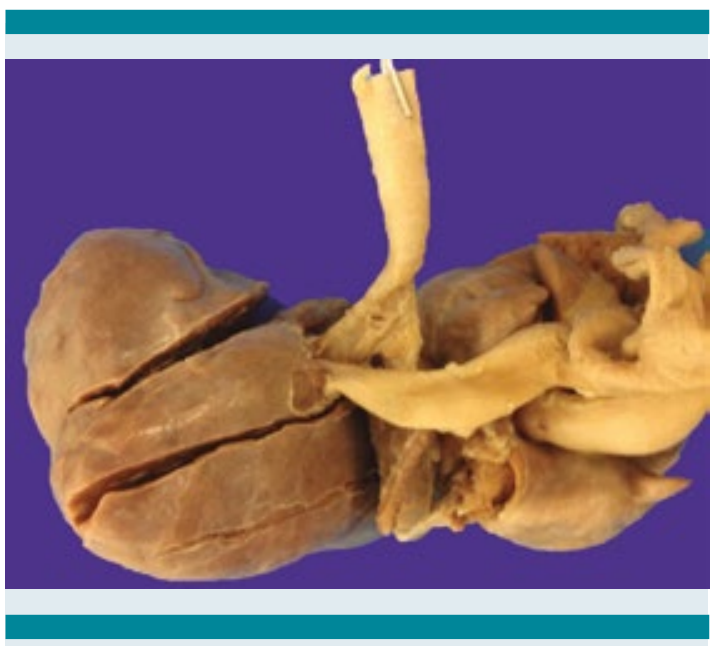

Figura 8. Vista posterior. La tráquea se estrecha en forma de embudo y a nivel de la carina se aprecia comprimida y aplanada por la arteria pulmonar izquierda retrotraqueal ("en hamaca").

El examen histológico de secciones cercanas al extremo superior de la tráquea mostró anillos cartilaginosos casi completos, sin la parte membranosa normal (Figura 9). A partir de la carina los bronquios se veían flácidos y aplanados, con un bronquio izquierdo más o menos normal y un bronquio derecho delgado y sin anillos cartilaginosos palpables. Había una marcada hipoplasia pulmonar derecha, con un pulmón de $3 \mathrm{~cm}$ de eje mayor y constaba de un solo lóbulo. El pulmón izquierdo fue normal salvo la lobulación anormal referida.

La gravedad de las anomalías en esta niña sugirió que no había estado tan asintomática como se refería antes de su ingreso. Siempre tuvo cianosis. Estaba muy baja de peso y talla, con una ventilación muy comprometida por la estenosis traqueal y una presión arterial pulmonar elevada. La depleción linfoide en timo, bazo y ganglios con involución aguda del timo sugieren un cuadro crónico con agudización final. En los pulmones se encontró una bronquiolitis extensa con focos neumónicos confluentes; probablemente fue el desencadenante de un giro hacia el agravamiento que condujo rápidamente a la muerte.

Las dos anomalías más significativas en esta niña: la arteria pulmonar izquierda aberrante con trayecto retrotraqueal, Ilamada arteria

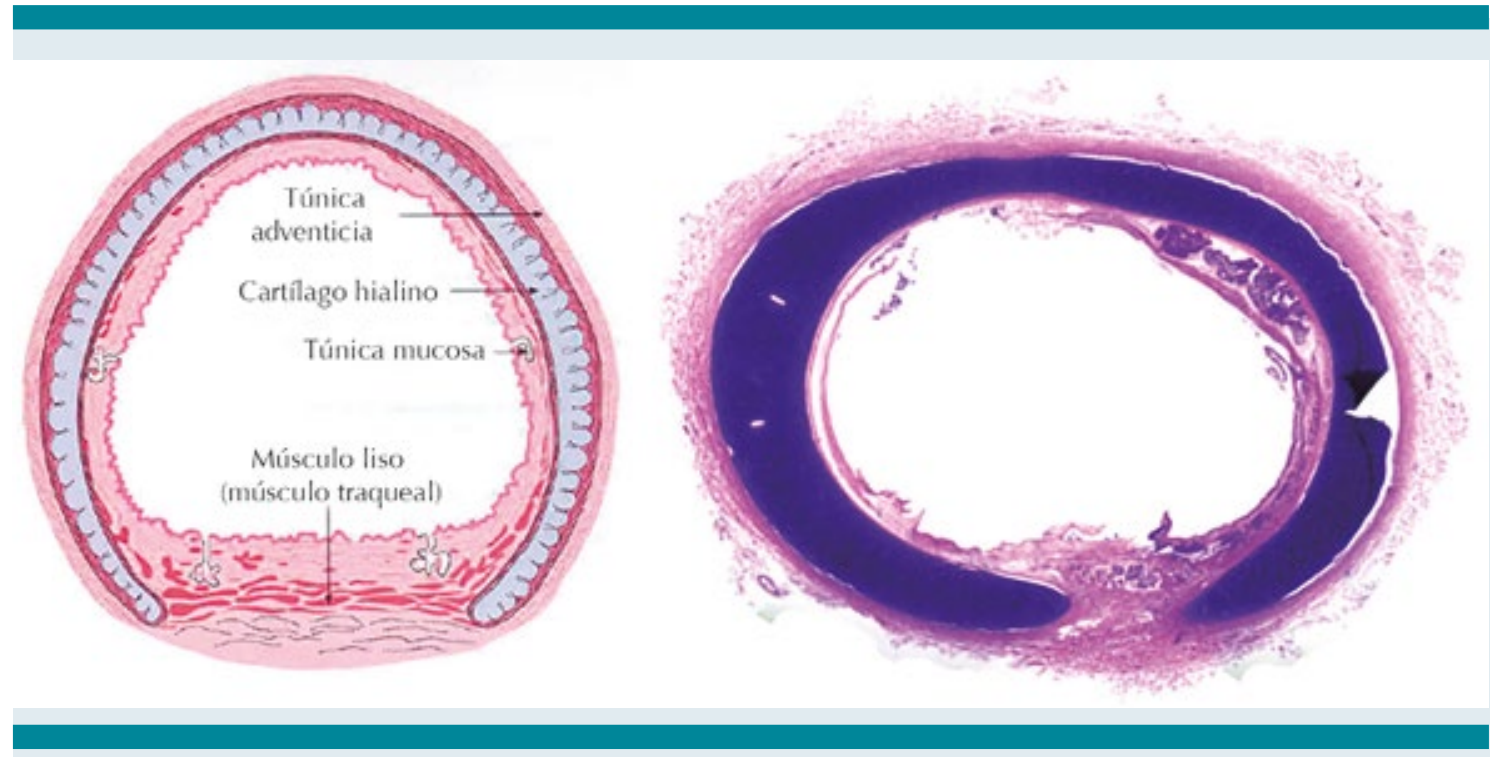

Figura 9. La tráquea estenótica muestra anillos cartilaginosos casi completos (esquema de tráquea normal.) 
pulmonar en hamaca (también llamada "sling pulmonar") y, por otro lado, la estenosis traqueal congénita con anillos cartilaginosos completos son malformaciones poco frecuentes, pero dentro su rareza, su asociación es reconocida y frecuente. ${ }^{1}$ La arteria pulmonar izquierda en hamaca, como malformación aislada, puede ser poco sintomática, ciertamente puede comprimir la tráquea pero su corrección quirúrgica es factible y su pronóstico es bueno. Cuando se asocia, como en nuestra paciente, a estenosis traqueal congénita de segmento largo, y esto sucede en más de la mitad de los casos de arteria pulmonar en hamaca, el cuadro clínico es mucho más complicado, la evolución desfavorable y la corrección quirúrgica difícil o imposible. ${ }^{2}$ Es importante recalcar que, si bien la arteria aberrante está comprimiendo la tráquea y produce una depresión evidente, la estenosis traqueal, con su estructura cartilaginosa anormal, es una alteración propia del desarrollo de la tráquea y no es secundaria a la compresión por la arteria anómala. La coincidencia de ambas anomalías evidentemente no es aleatoria y parece responder a defectos en la función diferenciadora y oganogénica del mesénquima cervical y se ubicarían en el espectro de los defectos del campo mesenquimal cervical. ${ }^{3}$ Es frecuente que pacientes con esta combinación de malformaciones tengan a su vez otras anomalías diversas, bronquiales, cardiovasculares, del intestino primitivo y otras. ${ }^{4}$

En nuestra paciente la microtia se notó desde el nacimiento. Se reconoce que puede ser una malformación aislada o formar parte del espectro óculo-aurículo vertebral. ${ }^{5}$ En nuestro caso el estudio completo de autopsia indicó que puede también ser un indicador de otras malformaciones, algunas que dominaron el cuadro clínico como la compleja anomalía cardiopulmonar, pero también otras que podrían haber pasado inadvertidas como el páncreas anular.

\section{A-14-21. DIAGNÓSTICOS ANATÓMICOS PRINCIPALES}

\section{Malformaciones múltiples}

Estenosis traqueal congénita de segmento largo, con cartílagos anormales.

Arteria pulmonar izquierda retrotraqueal (arteria en hamaca) con compresión traqueal extrínseca.

Pulmón derecho hipoplásico.

Cardiopatía congénita.

Conducto arterioso permeable.

Comunicación interventricular alta puntiforme.

Válvula tricúspide displásica.

Ausencia de vena cava inferior con hemiácigos.

\section{Microtia izquierda.}

Micrognatia.

Páncreas anular.

Bronquiolitis aguda con bronconeumonía multifocal.

\section{COMENTARIO CARDIOLÓGICO}

(Dra. Fabiola Pérez Juárez)

La arteria pulmonar en hamaca es una entidad muy rara, se asocia con estenosis bronquial y traqueal de manera frecuente. Aquí presentamos el caso de una niña de 3 meses con esta patología, estenosis traqueal e hipoplasia de pulmón derecho que se presentó con insuficiencia respiratoria grave. 
La arteria pulmonar en hamaca también conocida como "sling pulmonar" consiste en que la arteria pulmonar izquierda anormal se origina de la rama derecha de la arteria pulmonar, cruza entre la tráquea y el esófago y finalmente llega al pulmón izquierdo ${ }^{6}$ (Figura 10). En alrededor de $50-65 \%$ se puede encontrar un anillo traqueal completo y este se refiere como complejo "anillo-hamaca".,16

La embriologia del "sling pulmonar" no se ha documentado completamente. Algunos autores proponen que representa una falla en el desarrollo de la arteria pulmonar o bien que se origina cuando existe una conexión caudal del plexo post-bronquial izquierdo con la arteria pulmonar izquierda, resultando en que la arte-

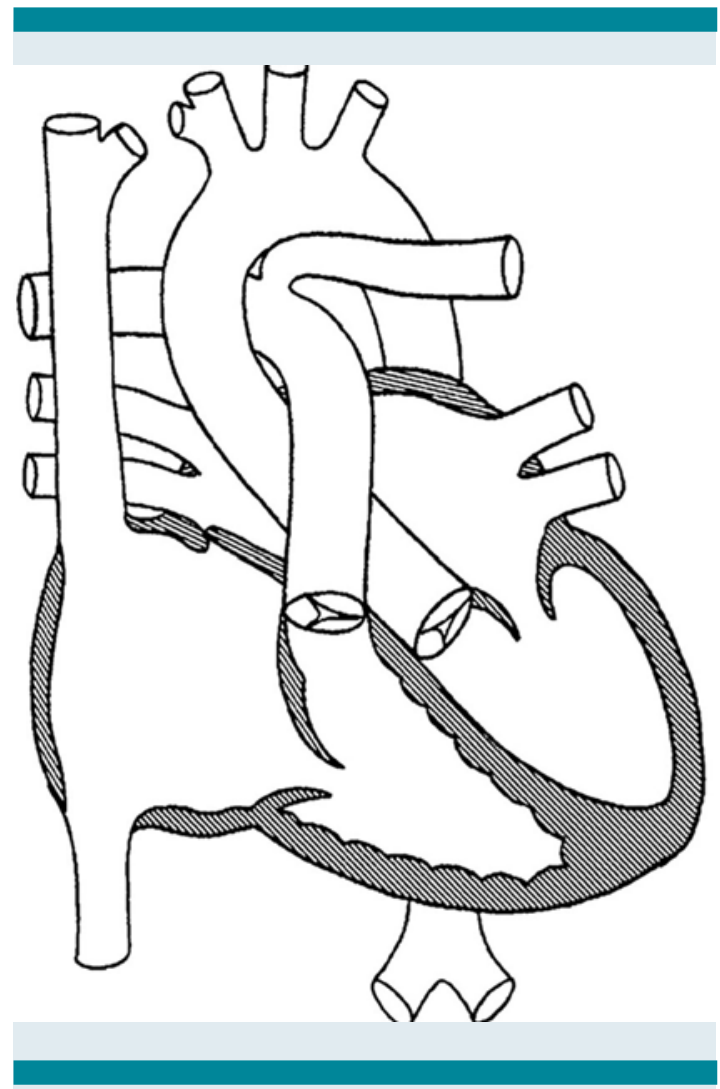

Figura 10. Dibujo que representa la rama pulmonar izquierda en hamaca. ria pulmonar izquierda cursa posterior al árbol traqueobronquial. ${ }^{7}$

En los casos reportados existe una relación de 3:1 hombres:mujeres y $90 \%$ de los pacientes tiene síntomas en el primer año de vida. Los signos atribuibles a insuficiencia respiratoria en estos pacientes puede ser secundario a compresión en la tráquea y pueden morir súbitamente por insuficiencia respiratoria grave.

Entre 40 y $50 \%$ de los pacientes con arteria pulmonar en hamaca tiene asociación con anomalías cardiovasculares mayores tal como conducto arterioso, comunicación interventricular, comunicación interatrial, vena cava superior izquierda y tetralogía de Fallot. Tal fue el caso de nuestra paciente donde se encontró un conducto arterioso con cortocircuito de derecha a izquierda, así como interrupción de la vena cava inferior. Es raro que los pacientes se compliquen por las anomalías cardiacas asociadas, las manifestaciones clínicas son secundarias a síntomas respiratorios casi siempre. Así que es fácil omitir el diagnóstico de cardiopatías asociadas en pacientes que muestran síntomas respiratorios sin cambios hemodinámicos, por lo que el ecocardiograma debe realizarse de manera rutinaria para descartar cardiopatías congénitas asociadas en pacientes con síntomas respiratorios inexplicables sin presencia de soplos, así como complementar el abordaje diagnóstico con angiotomografía computada, ya que juega un papel muy importante en el diagnóstico, no sólo para establecer de manera detallada las características de la rama izquierda anómala en hamaca, sino el desarrollo de la tráquea y la relación de ésta con la arteria pulmonar.,

Aunada a la arteria pulmonar en hamaca, la paciente presentó hipoplasia del pulmón derecho que también es una condición muy rara, se reporta una incidencia de 1:15,000 autopsias. 
Más de $50 \%$ de los pacientes mueren antes de los primeros 5 años de vida. ${ }^{9,10}$

El pronóstico de esta enfermedad depende de la presentación y de las patologías asociadas. En ausencia de otras malformaciones asociadas la rama izquierda en hamaca es compatible con la vida normal; sin embargo, una infección respiratoria grave durante la infancia es común en estos pacientes y puede llevar a neumonía y muerte.

Se piensa que los pacientes con esta patología y síntomas respiratorios moderados necesitan tratamiento quirúrgico y se han introducido diversas técnicas quirúrgicas. Sin embargo, a pesar de esta opción, la tasa de mortalidad es alta cuando existen anomalías asociadas. El pronóstico en pacientes asintomáticos es excelente y la cirugía no está indicada. Por lo que en pacientes lactantes con síntomas respiratorios recurrentes inexplicables es recomendable realizar un ecocardiograma como modalidad de primera opción para el diagnóstico, así como un estudio de angiotomografía de corazón y grandes vasos. No obstante, la condición de nuestro paciente fue muy grave y no fue posible completar el abordaje diagnóstico oportuno para poder plantear alguna posibilidad de tratamiento quirúrgico. ${ }^{11,12}$

\section{CONCLUSIÓN}

La utilidad de describir este caso no sólo es recordar la entidad de arteria pulmonar en hamaca e hipoplasia pulmonar como una de las posibles causas de patologías respiratorias graves, sino hacer énfasis en buscar patologías asociadas tal como estenosis traqueal y cardiopatías congénitas. Por lo que el diagnóstico oportuno y exacto permitirá brindar una atención completa y rápida y, tal vez, esto puede favorecer el pronóstico de futuros pacientes.

\section{REFERENCIAS}

1. Berdon WE, Baker DH Wung J-T. Complete cartilagering tracheal stenosis associated with anomalous left pulmonary artery: The ring-sling complex. Radiology. 1984;152:57-64.

2. Backer CL, Kelle AM, Mavroudis Cy otros. Tracheal reconstruction in children with unilateral lung agenesis or severe hypoplasia. Ann Torac Surg 2009;88:624-630.

3. Voland JR, Benirschke K, Saunders B. Congenital tracheal stenosis with associated cardiopulmonary anomalies: report of two cases with a review of the literature. Pediatr Pulmonol. 1986;2:247-249.

4. Medina-Escobedo G, López Corella E. Sling left pulmonary artery, bridging bronchus and associated anomalies. Am J Med Genet. 1992;44:303-306.

5. Llano Rivas I, González del Angel A, del Castillo V, Reyes R, Carnevale A. Microtia. A clinical and genetic study at the National Institute of Pediatrics in Mexico City. Arch Med Res. 1999;30:120-124.

6. Wu MH, Wu HY. Tracheobronchial stenosis caused by complex sling of left pulmonary and aorta. Ann Thorac Surg; 2016;102:e59.

7. Xie J, Juan YH, Wang Q, Chen J, ZhuangJ, Xie Z, et.al. Evaluation of left pulmonary artery sling, associated cardiovascular anomalies, and surgical outcomes using cardiovascular computed tomography angiography. Sci Rep 2017;5:4042-52.

8. Pierron C, Sigal-Cinqualbre A, Lambert V, Le Bret E.Left pulmonary artery sling with right lung aplasia. J Pediatr Surg. 2011;46:2190-4.

9. Wang JH1, Ding GC, Zhang MY, Liu M, Niu HY. Clinical and imaging features of pulmonary artery sling in infants without significant hemodynamic changes. Chin Med J. 2011;124:3412-4.

10. Dinamarco PVV, Ponce CC. Pulmonary agenesis and respiratory failure in childhood. Autopsy Case Rep 2015;5:29-32.

11. Ootaki Y, Yamaguchi M, Yoshimura N, Oka S. Pulmonary Agenesis with congenital heart disease. Pediatr Cardiol. 2004;25:145-8.

12. He H, Sun F, Ren W, Wang $Y, X u$ S. Diagnostic value of echocardiography in isolated congenital unilateral lung agenesis combined with different anomalies: Two rare cases in children. J Clin Ultrasound. 2016;44:514-7. 